\title{
The progress of family health nursing in remote and rural Scotland
}

\author{
Colin Macduff \\ Colin Macduff is Research Fellow, School of Nursing and Midwifery The Robert Gordon University,
} Aberdeen

Email:c.macduff@rgu.ac.uk

\begin{abstract}
I n 1998, the World Health Organization (WHO) Europe introduced the family health nurse (FHN) concept as a key part of the HEALTH 21 policy framework (WHO, 1998). The proposed role of this new community-based nurse was multifaceted and included helping individuals, families and communities to cope with illness and to improve their health. It was initially envisaged that 18 European countries would develop this new role through parallel processes of education and implementation, but in 2001 Scotland became the first country to initiate a pilot project.

Before the start of the project, the Scottish Executive Health Department (SEHD) summarized the principles of the new role as:

- A skilled generalist role encompassing a broad range of duties, dealing as the first point of contact with any issues that present themselves and referring on to specialists where a greater degree of expertise is required

- A model based on health rather than illness - the FHN would be expected to take a lead role in preventing illness and promoting health as well as caring for those members of the community who are ill and require nursing care
\end{abstract}

\section{ABSTRACT}

Since 2001 the World Health Organization Europe's family health nurse (FHN) role has been developing in remote and rural areas of Scotland. In 2003, an independent evaluation identified a need for facilitation of the FHN role and family-health orientated approaches with local primary health care teams. The Scottish Executive Health Department appointed three part-time, regionally-based family health practice development facilitators (FHPDFs) in December 2003 to work over an 18-month period. This article presents findings from a small study which sought these FHPDFs' judgements on individual FHN autonomy and supportive colleague action at 24 sites where FHNs were practising. These judgements reveal a picture of mixed progress that is consistent with findings from other related research. This collective overview is presented in the form of a new typology and the resultant implications for future development of family health nursing are discussed.

\section{KEY WORDS}

Family Health Nursing - Research • Practice development - Typology
- A role founded on the principle of caring for families rather than just the individuals within them.

- A concept of the nurse as first point of contact.

The SEHD saw this role as particularly suited to the needs of remote and rural communities and, accordingly, the pilot project took place in four remote and rural regions of Scotland from 2001-2003. During this time 31 FHNs were prepared (eleven in 2001 and 20 in 2002) through a degree level community specialist practitioner educational programme (Murray, 2004). These nurses all had extensive previous experience in community settings and 20 were also midwives. Nine already had a district nursing qualification and three already had a health visiting qualification. The new FHNs greatly valued the family health assessment/promotion skills learned on the educational programme, and these were seen as central to creating a distinctive new professional identity (Macduff and West, 2004).

An independent evaluation of the pilot project was also commissioned by the SEHD. This included evaluation of the operation and impact of family health nursing over the first year of practice (2002) at ten remote and rural Scottish primary health care sites. Full details of methods and findings are presented elsewhere (Macduff and West, 2003; Macduff and West, 2005) but, in summary, it was found that the FHN role was usually developed in a limited way on top of a district nursing caseload. All the new FHNs had returned to work at home bases where they had previously been working as community staff nurses or district nurses. Typically this new G-grade role was expected to contribute to the care of patients who were already a part of district nursing caseloads.

In this way the new role tended to supplement rather than supplant pre-existing community nursing activities. This supplementation usually took the form of in-depth assessment and intervention work with a small number of families, and health promotion work with groups in local communities. However, capacity to engage with whole families was found to be limited during the first year of practice. Similarly, commitment to service provision for those with illness made it difficult for the new FHNs to really take a lead role in promoting health in their local communities. There tended to be little change in terms of FHNs being first point of contact; some FHNs were necessarily the first point of contact, as there was no other type 
of nursing service immediately available (e.g. those working on small islands). Other FHNs would potentially be the first point of contact for their 'inherited' district nursing caseload patients and a small number of other families.

An initial typology of family health nursing emerged, which identified four distinct patterns of practice:

- High scope-slow build: Small, stable caseload with high pre-existing scope for nursing autonomy and practice development, but gradual introduction by FHN only, with little/no change in other professionals working practices

- Slow build-key ally: FHN role superimposed on moderate district nursing caseload within established and functional medium-sized PHCT. Gradual introduction by FHN with active, focused support from at least one other professional within the core PHCT

- Slow/No go: FHN role super-imposed on heavy district nursing caseload within established and functional medium sized PHCT, so only sporadic and limited introduction by FHN, with little/no change in other professionals working practices

- Bold build: Heavy district nursing caseload within established and functional medium sized PHCT, but FHN role not superimposed. New FHN caseload built vigorously through referrals from professionals and public.Autonomous workload management with high community outreach element. Some frictions at the boundaries of other professionals' roles. Tensions within the core PHCT.

This initial typology enabled the 2003 evaluation to address a subsequent question: what makes an FHN role work? The key factors to emerge were:

(i) The perceived scope and space to encourage implementing an FHN approach.

(ii) The local presence of at least one active supporter (key ally) who changes their own practice.

The presence of at least one of these factors seemed to be a necessary condition for progress. The drive and individual creativity of each FHN were also identified as inherently influential factors.

The final report of the evaluation (Macduff and West, 2003) suggested that there was a need for facilitation of the FHN role and family health-orientated approaches with local primary health care teams (PHCTs). This was swiftly enacted by the SEHD in December 2003 when they appointed three part-time, regionally-based family health practice development facilitators (FHPDFs) to work over an 18-month period. This article presents findings from a small research study which sought these FHPDFs' judgements on progress at 24 remote and rural sites where FHNs were practising during 2004.

\section{Design and methods}

By the end of 2004 each FHPDF had been in post for a year, during which time they had sought to facilitate FHN role development and family health-orientated approaches within the relevant PHCT sites in their own regions. This involved five sites in Orkney, eight sites in the Western Isles and eleven sites in the Highlands/Argyll and Clyde region.
Engagement with PHCTs usually took the form of regular site visits to meet team members and to facilitate review of working practices. This put the FHPDFs in a unique position to compare and contrast FHN practice development. As such, there seemed a good opportunity to seek their judgements on progress.

This was addressed by means of a short questionnaire, which was designed to build on the previous evaluation findings. As the new study sought the unique overview provided by the FHPDFs, there seemed opportunity to move on from the rather diffuse notion of perceived scope and space to encourage implementing an FHN approach, towards judgement on what family health nursing development had actually happened or been achieved. Here the concept of exercised FHN autonomy seemed more useful. As Hunt and Wainwright (1994) point out, autonomy in thinking, decision-making and acting is the key to role expansion in nursing. This concept also seemed useful in that it would include the influence of the individual FHN's own motivation.

The first factor that the FHPDFs were asked to rate was the level of autonomy actually being exercised to develop family health nursing practice. To do this they were asked to consider the extent to which FHN practice had determined its own priorities for action and then acted to deliver and sustain these. In this regard they were instructed to disregard whether the autonomy had been conferred (e.g. where an FHN had inherited substantial scope) or was inferred (e.g. where an FHN had interpreted the role in a specific way). The key criterion was the extent to which autonomy was actually being exercised to develop practice that was consistent with family health nursing, rather than any other professional discipline. A rating scale was developed so that they could indicate their judgement by putting a cross anywhere on a continuum line (Figure 1).

The other main question sought to move beyond the notion of key ally to achieve a broader picture of what colleague action had actually taken place. The literature on nursing role development (e.g. Tolson and West 1999) repeatedly emphasises that understanding and supportive action from professional colleagues is necessary for a new role to become integral and sustained in itself, and then influence the nature of wider service provision. Again detailed instructions were given to respondents in order to give a clear operational definition of colleague action. In this regard they were asked to rate the extent to which other professionals' had acted in order to support and develop a more family health orientated approach within the PHCT. It was emphasised that the key criteria here was

$\begin{array}{lclcl}\text { No FHN } & \text { Little FHN } & \text { Moderate } & \text { High FHN } & \text { Very high } \\ \text { autonomy } & \text { autonomy } & \text { FHN autonomy autonomy } & \text { FHN autonomy } \\ \text { exercised } & \text { exercised } & \text { exercised } & \text { exercised } & \text { exercised } \\ 0 & 1 & & & \end{array}$

Figure 1. Rating scale for FHN autonomy being exercised. 


$\begin{array}{lllll}\text { No } & \text { Little } & \text { Moderate } & \text { High } & \text { Very high } \\ \text { colleague } & \text { colleague } & \text { colleague } & \text { colleague } & \text { colleague } \\ \text { action } & \text { action } & \text { action } & \text { action } & \text { action } \\ 0 & 1 & & \end{array}$

Figure 2. Rating scale for colleague action to support a family health approach in the $\mathrm{PHCT}$

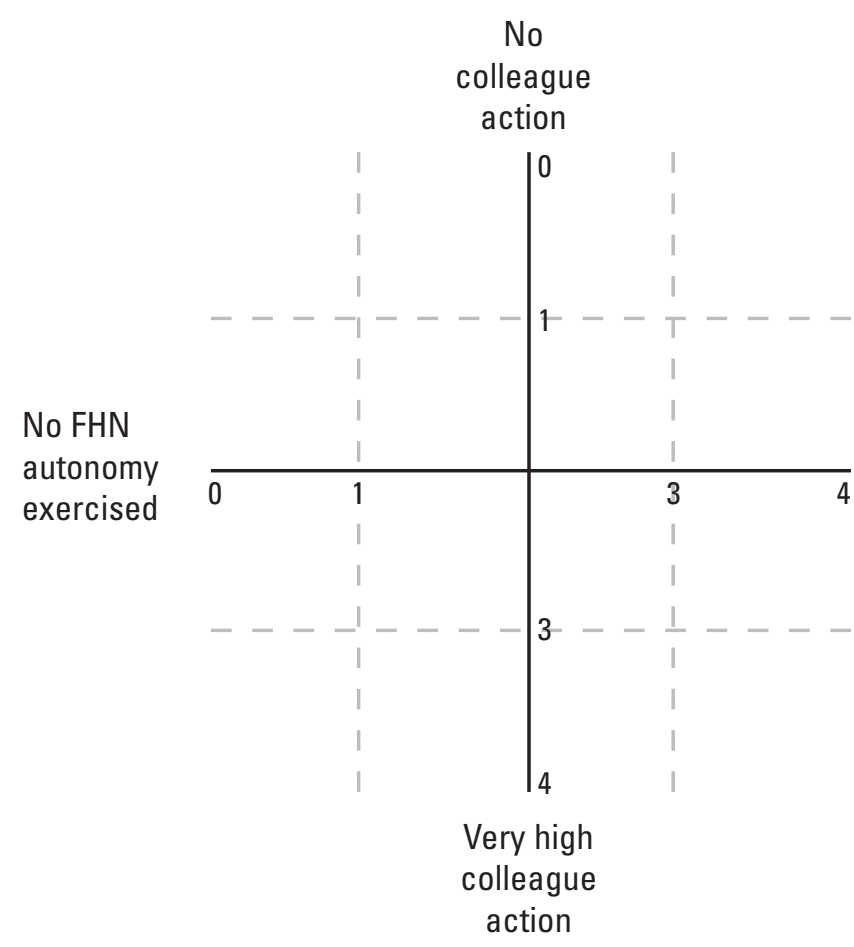

Figure 3. Matrix for mapping ratings of each FHN site.

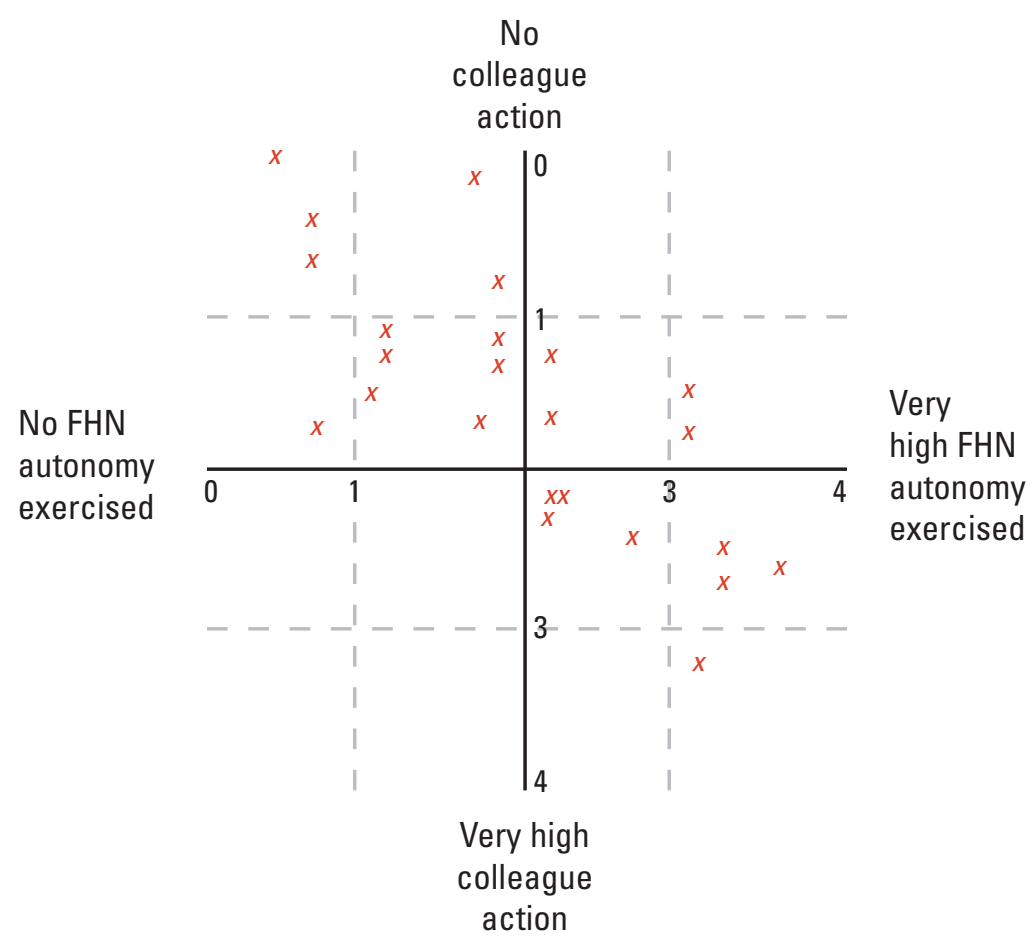

Figure 4. FHN site characteristics as rated by the FHPDFs ( $x=$ FHN site). the extent to which colleagues had recognized a need for a more family health orientated approach and then acted to make it a reality. This might range from no action, through change in working practice by one or two key allies, through to the successful enactment of a PHCTwide programme of activity. Again it was emphasised that the focus was on what had actually been achieved to date, rather than what might have been. A similar rating scale (Figure 2) was designed for this purpose.

The FHPDFs were then invited to map the two ratings they had made for each site onto a quartered matrix (Figure 3). In this way each site could be represented by a cross and a comparative mapping of site characteristics in their region could be visualised. They were also invited to comment on the process of making and mapping their judgements.

\section{Ethical considerations}

Ethical approval for the research was sought from each of the relevant NHS regional ethics committees and management approval for the research was sought from each of the relevant NHS health boards/divisions. In the letter inviting the FHPDFs to participate, they were assured that their judgements would be kept confidential by the researcher and that the reporting of findings would not name individual sites. Once all relevant approvals had been obtained, the questionnaires were sent out in December 2004.

\section{Findings}

All three FHPDFs responded to the questionnaire. Thus in aggregate their responses covered all 24 FHN sites that were active at the end of 2004. Their ratings of these sites in relation to the two main questions are collated in Figure 4.

As the diagonal trend in Figure 4 suggests, there was significant correlation between ratings of the degree of autonomous development of family health nursing practice and ratings of the degree of action colleagues had been taking to support and develop a more family orientated approach within the PHCT as a whole (Pearson coefficient $p=0.00$ ).

The FHPDFs' comments in relation to the process of making and mapping their judgements are summarized in Box 1.

\section{Discussion}

The results collated in Figure 4 provide a useful overview of the progress of family health nursing in remote and rural Scotland. Half of the sites are located in the upper left quadrant, signifying little or moderate practice development. The four sites in the upper right quadrant show moderate to high FHN autonomy in developing practice, but less active support from colleagues. The lower right quadrant shows four sites moving towards high FHN practice development and colleague action, and four that appear to be largely achieving this.

The correlation evident in the clustering of sites around the diagonal is perhaps not surprising, in that these were the perceptions of the FHPDFs who, during 
the preceding year, had invested much time and effort at local sites towards making such simultaneous development happen. In this regard it is notable that family-orientated colleague action only rose above a moderate level if, and when, FHN practice was substantively developed (i.e. the left lower quadrant in Figure 4 is empty). This would suggest that the FHNs and the FHPDFs were instrumental in driving forward family-orientated services at the sites where such services were becoming more developed. This was consistent with findings from a concurrent follow-up study which sought the perspectives of the FHNs and their professional colleagues (Macduff, 2005), and raised some concerns as to whether momentum would be sustained when the FHPDF contracts finished in May 2005.

Overall the picture of mixed progress that emerges from this study very much corroborates the findings from the concurrent follow-up study of other professionals. This is important to note, as there are inherent dangers in basing an overview on findings from three people who have been asked to make global judgements, a fact reflected in the concern that one FHPDF had about the subjective nature of the rating process. Moreover Box 1 shows one respondent's unease about the instruction to disregard underlying causes relating to FHN autonomy. The comment seems to be related to the focus on evaluative judgement rather than more in-depth exploration of perceptions, and this is acknowledged as a limitation of this small-scale study. Nevertheless, respondents' comments also show that the relative simplicity of this approach provided useful visualization, leading to clarification. It is interesting to note how one FHPDF's recognition of a pattern in her ratings of sites within her own region was later corroborated statistically when all FHPDF ratings were considered.

The quartered matrix also provides a useful framework for presenting the findings in typology format. Figure 5 suggests a new typology of family health nursing practice development, comprising five types.

The sites forming the upper left hand corner of Figure 5 may be termed 'no go', as there is typically neither enough FHN autonomy nor active colleague support to generate any substantive forward momentum. At these sites there has been a change in name to FHN but almost no change in individual nursing function or overall service delivery. The 'slow-build' types show somewhat more promise, but seem unlikely to develop substantively until FHN autonomy and colleague action both rise beyond moderate levels. The two sites at the right of the upper right quadrant show moderate-to-high FHN autonomy in developing practice, but less active support from colleagues. This can be characterized as a 'push-pull' pattern, in that typically the individual FHNs have been consistently active in pushing the autonomous development of their new role, but are still struggling against the pull exerted by the traditional role expectations of colleagues.

The lower right quadrant shows four sites moving towards high FHN practice development and colleague
Box 1. Summary of FHPDFs' comments on making and mapping judgements

'Autonomy is frequently outwith control of FHN and is not necessarily related to caseloads either. FHNs often spend time supporting social work services and this restricts scope for development.'

'Applying it has highlighted the fact that colleague action seems to be correlated with the level of autonomy.'

'Typology useful and has provided clarification.'

'Still very subjective in many ways. Would like to compare scores with others who perhaps know more or see it from a different perspective.'

action. This represents more significant and more balanced consolidation of family health nursing. As such, these sites are characteristic of a 'forming' pattern, whereby the respective FHNs are establishing a distinctive new approach that is valued and actively supported by colleagues (e.g. through appropriate referral of whole families). The four sites that are further towards the lower right hand corner of Figure 5 indicate progression from the forming pattern towards a 'transforming' pattern. The distinctive feature of the latter pattern seems to be a high level of active support from colleagues, that is enabling more substantive change to the nature of overall service provision (e.g. whereby colleagues' own practice has become more family health focused).

As Nolan et al (1998) note, typologies can provide useful ways of clarifying thinking. In this regard the types in Figure 5 suggest possibilities for moving on from the initial family health nursing typology, which related to practice at ten sites during the first year of role enactment. Indeed, the new typology may have wider relevance when considering other nursing role developments.

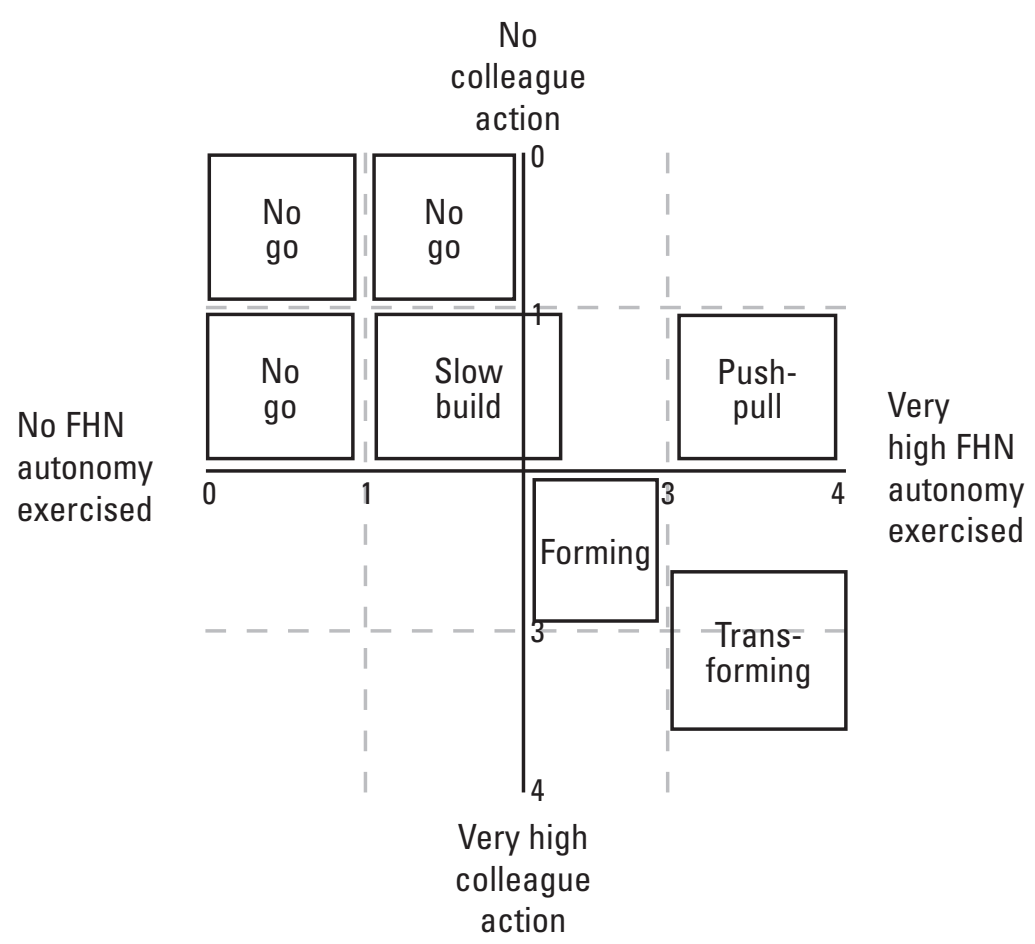

Figure 5. A new typology of family health nursing practice development 


\section{KEY POINTS}

- This study is part of the process of reviewing the development of the WHO Europe family health nurse concept in remote and rural Scotland. An overview was provided by a research study with three family health practice development facilitators.

- The study sought these facilitators' judgements on FHN autonomy and support from professional colleagues.

- Overall, the picture gained is of mixed progress.

- This study has led to the development of a new typology of family health nursing practice development.

\section{Conclusion}

The overview afforded by this study suggests that family health nursing is developing gradually in remote and rural areas of Scotland. As yet there are only a few sites where family health nursing is beginning to have a transforming influence on the overall nature of service delivery by the PHCT. In many ways this is not surprising, as the development to date has been relatively small scale and has taken place at a time when other significant developments have been impacting on the provision of remote and rural health care. Not least of these is the General Medical Services contract, which serves to facilitate reduction in GPs' out-of-hours commitments. Perhaps as importantly, from the point of view of family health nursing, the new GMS contract provides very little incentive for the PHCT to put whole families at the centre of health and social care provision. This makes it likely that FHNs will continue to experience tensions among the many elements of their wide-ranging remit. In particular, they are likely to feel tension between the aspiration to focus on family health and the obligation to service a primary care system that is predicated on the provision of care to individuals.

Beyond the remote and rural context, a small urban pilot of family health nursing is currently taking place in Glasgow. Findings from this project are due to be published in 2006 Moreover, an evaluation report on the progress of family health nursing within Europe is also due in 2006. In this way a broader overview should be available to complement the one provided by this study.

BJCN

Hunt G, Wainwright P, eds (1994) Expanding the Role of the Nurse. Blackwell, Oxford

Macduff C (2005) A follow-up study of professionals' perspectives on the development of family health nursing. The Robert Gordon University, Aberdeen

Macduff C, West B (2003) Evaluating Family Health Nursing through education and practice. Scottish Executive Social Research, The Stationery Office, Edinburgh

Macduff C, West B (2004) An evaluation of an educational programme to prepare family health nurses. Nurse Educ Today 24(7): 575-83

Macduff C, West B (2005) An evaluation of the first year of family health nursing practice in Scotland. Int J Nurs Stud 42(1): 47-59

Murray I (2004) Family Health Nursing: the education programme for the WHO Europe Scottish Pilot. Br J Community Nurs 9(6): 245-50

Nolan M, Keady J, Grant G (1995) Developing a typology of family care: implications for nurses and other service providers. J Adv Nurs 21(2): 256-65

Tolson D, West B (1999) An exploration of role development in nursing and midwifery: Scotland. Scottish Executive Stationery Office, Edinburgh

World Health Organization (1998) HEALTH 21: the health for all policy framework for the WHO European Region (European Health for All Series No. 5).WHO Regional Office for Europe, Copenhagen

\section{Palliative Care for the Primary Care Team}

\section{by Eileen Palmer and John Howarth}

ISBN: 185642229 1; 234 x 156 mm; p/back; 320 pages; publication April 2005; £32.99

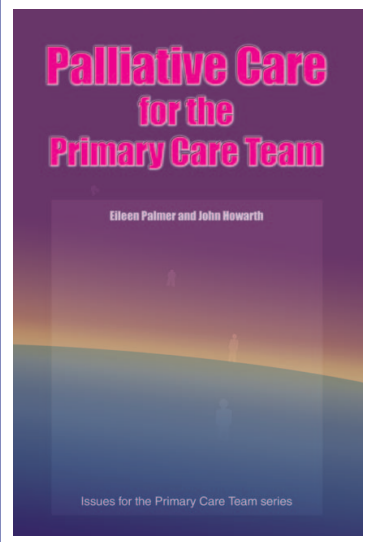

Ninety per cent of palliative care is done in primary care and most patients, given the choice, would prefer to die in their own home. The content of this new book makes few assumptions about existing knowledge, but is designed as a practical handbook for busy GPs and other members of the primary care team. The text is based around five core themes, which run throughout. Namely: the importance and value of the basic therapeutic relationship between patient and health worker; the principle of dealing with the patient, not in isolation, but as part of a family and community; the importance of whole person care; good palliative care is pro-active; and good communication is essential. Palliative care rarely goes wrong due to a lack of knowledge about subgroups of morphine receptors in the central nervous system. It is usually because of failure in one of these five basic areas. This is the challenge for primary care: a challenge that this book addresses and meets. Eileen Palmer is Medical Director to West Cumbria Hospice at Home and a Consultant in Palliative Medicine to North Cumbria Primary Care Trust and North Cumbria Acute Trust. John Howarth is a General Practitioner in Cumbria, and former Medical Advisor to West Cumbria Hospice at Home.

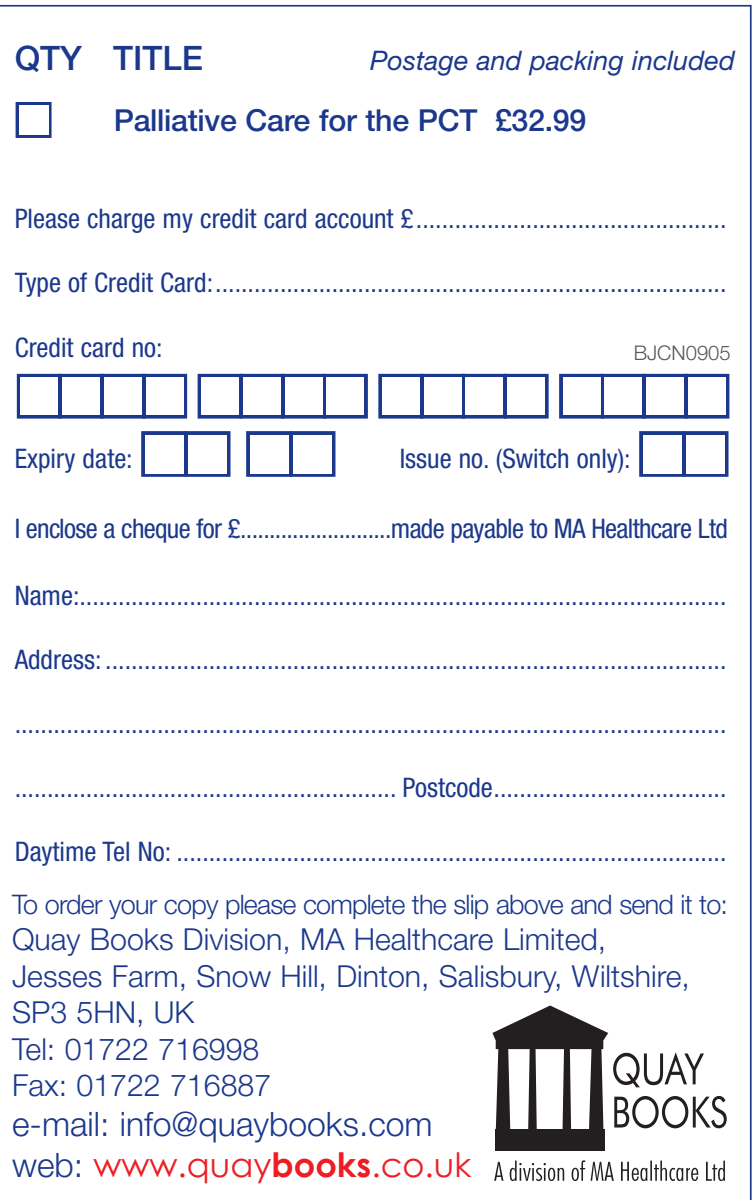

